\title{
Totally Real Submanifolds of a Quaternion Projective Space (*).
}

\author{
BaNg-Yen Chen (1) - ChORNG-ShI Houh (East Lansing, Michigan, U.S.A.)
}

Dedicated to Professor T. Otsuki on his sixtieth birthday

Summary. - See Introduction.

\section{1. - Introduction.}

A quaternion manifold is defined as a Riemannian manifold whose holonomy group is a subgroup of $S p(1) \cdot S p(m)$. The quaternion projective space $P^{m}(\boldsymbol{H})$ and its noncompact dual are two important examples of quaternion manifolds. It is well-known that they have constant positive and negative quaternion sectional curvature, respectively. We simply call quaternion manifolds with constant quaternion sectional curvature the quaternion-space-forms.

Let $N$ be a submanifold of a quaternion manifold $M . N$ is called an invariant submanifold of $M$ if $N$ is invariant under the curvature operator of $M$, i.e., $\widetilde{R}(X, Y)$. $\cdot(T N) \subset T N$ for all vector fields $X, Y$ in $T N$, where $T N$ is the tangent bundle of $N$ and $\tilde{R}$ is the curvature tensor of $M$. In [1], it is proved that if $N$ is an $n$-dimensional $(n \geqq 4)$ invariant submanifold of a quaternion-space-form of nonzero quaternion sectional curvature, then $N$ is either a totally real manifold or a half-quaternion submanifold, or a quaternion submanifold.

In this paper we shall study some fundamental properties of totally real submanifolds in a quaternion-space-form. The other submanifolds will be studied in another paper.

In $\S 2$, some fundamental formulas and properties of totally real submanifolds will be obtained.

In $\S 3$, we shall establish a sufficient condition for a totally real submanifold in a quaternion projective space $P^{m}(H)$ to be totally geodesic in terms of scalar curvature.

In $\S 4$, some pinching theorems on sectional curvature for totally real submanifolds in $\boldsymbol{P}^{m}(\boldsymbol{H})$ and $\boldsymbol{P}^{m}(\boldsymbol{C})$ are obtained. Some of these results are independent of codimension.

In the last section, we shall study minimal totally real immersion of real-spaceforms in quaternion-space-form.

(*) Entrata in Redazione il 19 ottobre 1977.

(1) Partially supported by NSF under Grant MCS 76-06318. 


\section{2. - Totally real submanifolds.}

Let $M$ be a $4 m$-dimensional quaternion manifold with metric $g$. Then there exists a 3-dimensional vector space $V$ of tensors of type $(1,1)$ with local basis of almost Hermitian structures $I, J, K$ such that

(a) $I J=-J I=K, J K=-K J=I, K I=-I K=J, I^{2}=J^{2}=K^{2}=-1$,

(b) for any local cross-section $\varphi$ of $V, \nabla_{x} \varphi$ is also a cross-section of $V$, where $X$ is an arbitrary vector field in $M$ and $\nabla$ the Riemannian-connection on $M$.

Condition $(b)$ is equivalent to the following condition:

$(b)^{\prime}$ there exist local 1-forms $p, q$ and $r$ such that

$$
\begin{aligned}
& \nabla_{X} I=r(X) J-q(X) K, \\
& \nabla_{X} J=-r(X) I+p(X) K, \\
& \nabla_{X} K=q(X) I-p(X) J .
\end{aligned}
$$

Let $X$ be anit vector on the quaternion manifold $M$. Then $X, I X, J X$ and $K X$ form an orthonormal frame on $M$. We denote by $Q(X)$ the 4-plane spanned by them. We call $Q(X)$ the quaternion-section determined by $X$. For any two vectors $X, Y$ in $M$, if $Q(X)$ and $Q(X)$ are orthogonal, the plane $\pi(X, Y)$ spanned by $X, Y$ is called a totally real plane. Any 2-plane in a quaternion-section is called a quaternion-plane. The sectional eurvature of a quaternion-plane $\pi$ is called the quaternion sectional curvature of $\pi$. A quaternion manifold is a quaternion-space-form if its quaternion sectional curvatures are equal to a constant $e$.

It is well-known that a quaternion manifold $M$ is a quaternion-space-form if and only if its curvature tensor $\tilde{R}$ is of the following form [4]:

$$
\begin{aligned}
\tilde{R}(X, Y) Z= & \frac{e}{4}\{g(Y, Z) X-g(X, Z) Y+g(I Y, Z) I X-g(I X, Z) I Y \\
& +2 g(X, I Y) I Z+g(J Y, Z) J X-g(J X, Z) J Y+2 g(X, J Y) J Z \\
& +g(K Y, Z) K X-g(K X, Z) K Y+2 g(X, K Y) K Z\},
\end{aligned}
$$

for vector fields tangent to $M$.

In the following, let $M^{m}(c)$ denote a $4 m$-dimensional quaternion-space-form of quaternion sectional curvature $e$, and let $P^{m}(H)$ denote the $4 m$-dimensional quaternion projective space of constant quaternion sectional eurvature $\theta>0$.

Let $N$ be an $n$-dimensional Riemannian manifold and $x: N \rightarrow M^{m}(c)$ be an isometric immersion of $N$ into a quaternion manifold $M^{m}(c)$. We call $N$ a totally real 
submanifold of $M^{m}(c)$ if each tangent 2-plane of $N$ is mapped by $x$ into a totally real plane in $M^{m}(c)$.

Let $\nabla$ and $\tilde{\nabla}$ be the Riemannian connections on $N$ and $M^{m}(e)$, respectively. The second fundamental form $\sigma$ of the immersion is given by $\sigma(X, Y)=\widetilde{\nabla}_{X} Y-\nabla_{X} Y$, where $X, Y$ are vector fields tangent to $N$ and $\sigma$ is a normal-bundle-valued symmetric 2 -form on $N$. For a normal vector field $\xi$ on $N$, we write $\widetilde{\nabla}_{x} \xi=-A_{\xi} X+D_{X} \xi$, where $-A_{\xi} X$ (respectively, $D_{x} \xi$ ) is the tangential (respectively, normal) component of $\tilde{\nabla}_{X} \xi$. We have $g(\sigma(X, Y), \xi)=g\left(A_{\xi} X, Y\right)$.

The mean-curvature vector $H$ is defined by $H=$ trace $\sigma / n . N$ is said to be minimal if $H=0$ identically. For the second fundamental form $\sigma$, we define the covariant differentiation $\bar{\nabla}$ with respect to the connection in the (tangent bundle) $\oplus$ (normal bundle) by

$$
\left(\bar{\nabla}_{X} \sigma\right)(Y, Z)=D_{X}(\sigma(Y, Z))-\sigma\left(\nabla_{X} Y, Z\right)-\sigma\left(Y, \nabla_{X} Z\right)
$$

for all vector fields $X, Y, Z$ tangent to $N$.

Let $R$ be the curvature tensor of $N$. Then the equation of Gauss is given by

$$
\begin{aligned}
g(R(X, Y) Z, W)=g(\tilde{R}(X, Y) Z, W)+g(\sigma(X, W), \alpha(X, Z)) & \\
& -g(\sigma(X, Z), \sigma(Y, W)),
\end{aligned}
$$

for $X, Y, Z, W$ tangent to $N$.

Assume that $N$ is a totally real submanifold of $M$. For any orthogonal vectors $X$, $Y$ in $N, \pi(X, Y)$ is totally real in $M^{m}(c)$. Hence, $Q(X)$ and $Q(Y)$ are also orthogonal. We thus have $g(X, \varphi \bar{Y})=g(\psi X, Y)=0$ for $\varphi, \psi=I$, $J$, or $K$. By (2.2) we have

$$
\tilde{R}(X, Y) Z=\frac{e}{4}\{g(Y, Z) X-g(X, Z) Y\},
$$

for $X, Y, Z \in T N$. Hence (2.4) reduces to

$$
\begin{aligned}
g(R(X, Y) Z, W)=\frac{c}{4}\{g(Y, Z) & g(X, W)-g(X, Z) g(Y, W)\} \\
& +g(\sigma(X, Z), \sigma(X, W))-g(\sigma(X, Z), \sigma(Y, W))
\end{aligned}
$$

Since $\operatorname{dim} N=n$ and $N$ is totally real in $M^{m}(e)$, we have $n \leqq m$. Let $p=4 m-n$. In $M^{m}(e)$ we choose a local field of orthonormal frames:

$$
\begin{array}{ll}
e_{1}, \ldots, e_{m}, e_{n+1}, \ldots, e_{m} ; & e_{I(1)}=I e_{1}, \ldots, e_{I(m)}=I e_{m} \\
e_{J(1)}=J e_{1}, \ldots, e_{J(m)}=J e_{m} ; & e_{K(1)}=K e_{1}, \ldots, e_{K(m)}=K e_{m},
\end{array}
$$

in such a way that, restricted to $N, e_{1}, \ldots, e_{n}$ are tangent to $N$. With respect to this 
frame field, $I, J, K$ have the following forms;

$$
I=\left(\begin{array}{cccc}
0 & -E & 0 & 0 \\
E & 0 & 0 & 0 \\
0 & 0 & 0 & -E \\
0 & 0 & E & 0
\end{array}\right), \quad J=\left(\begin{array}{cccc}
0 & 0 & -E & 0 \\
0 & 0 & 0 & E \\
E & 0 & 0 & 0 \\
0 & -E & 0 & 0
\end{array}\right),
$$

$$
K=\left(\begin{array}{cccc}
0 & 0 & 0 & -E \\
0 & 0 & -E & 0 \\
0 & E & 0 & 0 \\
E & 0 & 0 & 0
\end{array}\right)
$$

where $E$ denotes the $(m \times m)$-identity matrix

We shall use the following convention on the range of indices unless otherwise stated:

$$
\begin{aligned}
& A, B, C, D=1, \ldots, m, I(1), \ldots, I(m), J(1), \ldots, J(m), K(1), \ldots, K(m), \\
& k, i, j, k, I=1, \ldots, n, \\
& \alpha, \beta=n+1, \ldots, m, I(1), \ldots, K(m), \\
& \lambda, \mu=n+1, \ldots, m . \\
& \varphi, \psi=I, J, K .
\end{aligned}
$$

With respect to the frame field of $M^{m}(c)$ chosen above, let

$$
\omega^{1}, \ldots, \omega^{n}, \omega^{n+1}, \ldots, \omega^{n n}, \omega^{T(1)}, \ldots, \omega^{I(m)}, \omega^{J(1)}, \ldots, \omega^{J(m)}, \omega^{K(1)}, \ldots, \omega^{K(m)}
$$

be the field of dual frames. Then the structure equations of $M^{m}(c)$ are given by

$$
\begin{array}{ll}
d \omega^{A}=-\sum \omega_{B}^{A} \wedge \omega^{B}, & \omega_{B}^{A}+\omega_{A}^{B}=0 \\
d \omega_{B}^{A}=-\sum \omega_{C}^{A} \wedge \omega_{B}^{C}+\widetilde{\Omega}_{B}^{A}, & \widetilde{\Omega}_{B}^{A}=\frac{1}{2} \sum \widetilde{R}_{A B O D} \omega^{C} \wedge \omega^{D} .
\end{array}
$$

Since $N$ is totally real, $Q\left(e_{i}\right)$ and $Q\left(e_{j}\right), i \neq j$ are orthogonal. Thus, $g\left(\varphi\left(e_{i}\right), \psi\left(e_{j}\right)\right)=0$ for $i \neq j$. From these and condition (2.1) for $M^{m}(c)$, we have

$$
\begin{array}{lll}
\omega_{j}^{i}+\omega_{i}^{j}=0, & \omega_{j}^{i}=\omega_{\varphi(j)}^{\varphi(i)}, & \omega_{j}^{q(i)}=\omega_{i}^{\varphi(j)} \\
\omega_{\mu}^{\lambda}+\omega_{\lambda}^{\mu}=0, & \omega_{\mu}^{\lambda}=\omega_{\varphi(\mu)}^{\varphi(\lambda)}, & \omega_{\mu}^{\varphi(\lambda)}=\omega_{\lambda}^{q(\mu)}, \\
\omega_{\lambda}^{i}+\omega_{i}^{\lambda}=0, & \omega_{\lambda}^{i}=\omega_{\varphi(\lambda)}^{\varphi(i)}, & \omega_{\lambda}^{\varphi(i)}=\omega_{i}^{q(\lambda)} .
\end{array}
$$


BANG-YeN CHEN - CHORNG-SHI HouH: Totally real submanifolds, eto. 189

Restricting these forms to $N$ we have the following structure equations of the immersion:

$$
\begin{aligned}
& \omega^{\alpha}=\mathbf{0}, \\
& d \omega_{j}^{i}=-\sum \omega_{k}^{i} \wedge \omega_{j}^{k}+\Omega_{j}^{i}, \quad \Omega_{j}^{i}=\frac{1}{2} \sum R_{i j k l} \omega^{k} \wedge \omega^{l}, \\
& \Omega_{j}^{i}=\Omega_{j}^{i}-\sum \omega_{\alpha}^{i} \wedge \omega_{j}^{\alpha} \quad \text { (the equation of Gauss) } .
\end{aligned}
$$

If we write $\omega_{i}^{\alpha}=\sum h_{i j}^{\alpha} \omega^{j}$, then

$$
h_{i j}^{\alpha}=h_{j i}^{\alpha}, \quad h_{i j}^{\alpha}=g\left(A_{\alpha} e_{i}, e_{i j}\right),
$$

where $A_{\alpha}=A_{e_{\alpha}}$ and the equation $(2.4)^{\prime}$ of Gauss is written as

$$
R_{i j k l}=\tilde{R}_{i j k l}+\sum\left(h_{i k}^{\alpha} h_{j l}^{\alpha}-h_{i l}^{\alpha} h_{j k}^{\alpha}\right)
$$

Moreover, from (2.7), we have

$$
h_{j k}^{\varphi(i)}=h_{i k}^{\varphi(j)}=h_{j i}^{\varphi(k)}
$$

If the second fundamental tensors commute, i.e., $\left[A_{\xi}, A_{\eta}\right]=0$ for any vector fields $\xi$, $\eta$ normal to $N$. Then, by a suitable choise of $e_{1}, \ldots, e_{n}$, we have $h_{j k}^{\varphi(i)}=0$ for $j \neq k$. Thus, by using (2.9), we find

$$
h_{j k}^{\varphi(i)}=0 \quad \text { for } i \neq j \text { or } j \neq k .
$$

In particular, if $n=m,(2.4)^{\prime}$ and $(2.10)$ imply the following.

Proposition 1. - Let $N$ be an n-dimensional totally real submanifold of a quaternion-space-form $M^{n}(c)$. If the second fundamental tensors commute, then $N$ is of constant sectional curvature c/4.

If $N$ is totally umbilical and totally real, the second fundamental tensors commute. Thus Proposition 1 implies the following.

COROLLARY 1. - Let $N$ be an n-dimensional totally umbilical and totally real submanifold of $M^{n}(c)$. Then $N$ is totally geodesic and of constant sectional curvature $c / 4$.

With respect to the frame fields chosen above, the curvature tensor $\tilde{R}$ of (1.2) is written as

$$
\begin{aligned}
\tilde{R}_{A B C D}= & c / 4\left(\delta_{A C} \delta_{B D}-\delta_{A D} \delta_{B C}+I_{A C} I_{B D}-I_{A D} I_{B C}+2 I_{A B} I_{C D}\right. \\
& \left.+J_{A C} J_{B D}-J_{A D} J_{B C}+2 J_{A B} J_{C D}+K_{A C} K_{B D}-K_{A D} K_{B C}+2 K_{A B} K_{C D}\right),
\end{aligned}
$$

where $\delta_{A B}$ is the Kronecker delta. 
By the equation $(2.4)^{\prime}$ of Gauss, the sectional curvature $K(X, Y)$ of $N$ for a plane determined by orthonormal vectors $X, Y$ is given by

$$
\Pi(X, Y)=g(R(X, Y) Y, X)=\frac{e}{4}+\sum\left\{g\left(A_{\alpha} X, X\right) g\left(A_{\alpha} Y, Y\right)-g\left(A_{\alpha} X, Y\right)^{2}\right\}
$$

The Ricei tensor $S$ of $N$ is given by

$$
\begin{aligned}
S(X, Y)=\sum g\left(R\left(e_{i}, X\right) Y, e_{i}\right) & \\
& =\frac{(n-1) c}{4} g(X, Y)+\sum\left\{\left(\operatorname{tr} A_{\alpha}\right) g\left(A_{\alpha} X, Y\right)-g\left(A_{\alpha} X, A_{\alpha} Y\right)^{2}\right\},
\end{aligned}
$$

and the scalar curvature $\varrho$ of $N$ is given by

$$
\varrho=\frac{n(n-1)}{4} c+\sum\left(\operatorname{tr} A_{\alpha}\right)^{2}-\|\sigma\|^{2},
$$

where $\left\|\sigma^{2}\right\|=\sum h_{i j}^{\alpha} h_{i j}^{\alpha}$ : In particular, if $N$ is minimal, then

$$
\begin{aligned}
& S-\frac{1}{4}(n-1) \operatorname{lo} \quad \text { is negative semidefinite, } \\
& \varrho \leqq \frac{1}{4}(n-1) n c .
\end{aligned}
$$

As an immediate consequence of these relations, we have the following characterizations of totally real, totally geodesic submanifolds.

Proposimion 2. - Let $N$ be an $n$-dimensional totally real minimal submanifold of a quaternion-space-form $M^{m}(c)$. Then $N$ is totally geodesic if and only if it satisfies one of the following conditions:
(a) $K=\frac{e}{4}$,
(b) $S=\frac{1}{4}(n-1) e g,(c)$
$\varrho=\frac{1}{4} n(n-1) e$

Reicark 1. - Since an $m$-dimensional real projective space $\boldsymbol{P}^{m}(\boldsymbol{R})$ of constant sectional curvature $o / 4$ can be isometrically imbedded in the $4 m$-dimensional quaternion projective space $\boldsymbol{P}^{m}(\boldsymbol{H})$ of quaternion sectional curvature $c$ as a totally real totally geodesic submanifold, every submanifold $N$ of $\boldsymbol{P}^{m}(\boldsymbol{R})$ can be regarded as a totally real submanifold of $P^{m}(\boldsymbol{H})$. If $N$ is minimal in $P^{m}(\boldsymbol{R}), N$ is a totally real, minimal submanifold in $\boldsymbol{P}^{n}(\boldsymbol{H})$.

Since there exist totally umbilical hypersurfaces in $P^{n+1}(\boldsymbol{R})$ which is not totally geodesic, there exist $n$-dimensional totally umbilical, totally real submanifold in $P^{n+1}(\boldsymbol{H})$ which is not totally geodesic in $P^{n+1}(\boldsymbol{H})$. Thus the codimensions in Proposition 1 and Corollary 1 are best possible. 


\section{3. - Pinchings on scalar curvature.}

In [3], the Laplacian of $\|\sigma\|^{2}$ was calculated for a minimal submanifold in a locally symmetric manifold, i.e., the following formula holds:

$$
\begin{aligned}
\frac{1}{2} \Delta\|\sigma\|^{2}=\left\|\bar{\nabla} \sigma^{\alpha}\right\|^{2}+ & \sum \operatorname{tr}\left(A_{\alpha} A_{\beta}-A_{\beta} A_{\alpha}\right)^{2}-\sum\left(\operatorname{tr} A_{A} A_{\beta}\right)^{2} \\
& +\sum\left(4 \tilde{R}_{\alpha \beta i j} h_{j k}^{\alpha} h_{i k}^{\alpha}-\tilde{R}_{\alpha k \beta k} h_{i j}^{\alpha} h_{i j}^{\beta}+2 \widetilde{R}_{i j k j} h_{i j}^{\alpha} h_{k l}^{\alpha}+2 \tilde{R}_{i j k l} h_{i l}^{\alpha} h_{i k}^{\alpha}\right) .
\end{aligned}
$$

Since $N$ is minimal, (2.5) and (3.1) imply

$$
\begin{gathered}
4 \tilde{R}_{\alpha \beta i j} h_{j k}^{\alpha} h_{i k}^{\beta}=c \sum_{i, \varphi} \operatorname{tr} A_{\varphi(i)}^{2}, \\
\tilde{R}_{\alpha k \beta k} h_{i j}^{\alpha} h_{i j}^{\beta}=\frac{n c}{4}\|\sigma\|^{2}+\frac{3}{4} c \sum_{i, \omega} \operatorname{tr} A_{\varphi(i)}^{2}, \\
\tilde{R}_{i j k j} h_{i l}^{\alpha} h_{l l l}^{\alpha}=\frac{1}{4}(n-1) c\|\sigma\|^{2}, \\
\tilde{R}_{i j k l} h_{i l}^{\alpha} h_{j k}^{\alpha}=\frac{o}{4}\|\sigma\|^{2} .
\end{gathered}
$$

Moreover, by using (2.8) we find

$$
\begin{aligned}
\sum\left(\tilde{R}_{i j k j} h_{i l}^{\alpha} h_{k l}^{\alpha}+\tilde{R}_{i j k l} h_{i l}^{\alpha} h_{j k}^{\alpha}\right)=\sum\left(R_{i j k j} h_{i l}^{\alpha} h_{k l}^{\alpha}+R_{i j k l} h_{k l}^{\alpha} h_{j l k}^{\alpha}\right) & \\
& -\frac{1}{2} \sum \operatorname{tr}\left(A_{\alpha} A_{\beta}-A_{\beta} A_{\alpha}\right)^{2}+\sum\left(\operatorname{tr} A_{\alpha} A_{\beta}\right)^{2} .
\end{aligned}
$$

Combining (3.4), (3.5) and (3.6), we obtain

$$
\begin{aligned}
\sum\left(R_{i j k j} h_{i l}^{\alpha} h_{k l}^{\alpha}+R_{i j k l} h_{i l}^{\alpha} h_{j k}^{\alpha}\right)= & \frac{1}{2} \sum \operatorname{tr}\left(A_{\alpha} A_{\beta}-A_{\beta} A_{\alpha}\right)^{2} \\
& -\sum\left(\operatorname{tr} A_{\alpha} \cdot A_{\beta}\right)^{2}+\frac{n c}{4}\|\sigma\|^{2}
\end{aligned}
$$

Substituting (3.2), (3.3), (3.4) and (3.5) into (3.1) we obtain

$$
\begin{aligned}
\frac{1}{2} \Delta\|\sigma\|^{2}=\|\bar{\nabla} \sigma\|^{2}+\sum \operatorname{tr}\left(A_{\alpha} A_{\beta}-A_{\beta} A_{\alpha}\right)^{2}-\sum( & \left.\operatorname{tr} A_{\alpha} A_{\beta}\right)^{2} \\
& +\frac{n c}{4}\|\sigma\|^{2}+\frac{e}{4} \sum_{i, \varphi}\left(\operatorname{tr} A_{\varphi(i)}^{2}\right) .
\end{aligned}
$$

On the other hand, by (3.1)-(3.7), we also find

$$
\begin{aligned}
\frac{1}{2} \Delta\|\sigma\|^{2}=\|\bar{\nabla} \sigma\|^{2}+\frac{1-a}{2} \sum \operatorname{tr}\left(A_{\alpha} A_{\beta}-\right. & \left.A_{\beta} A_{\alpha}\right)^{2}+a \sum\left(\operatorname{tr} A_{\alpha} A_{\beta}\right)^{2} \\
& -\frac{n a \sigma}{4}\|\sigma\|^{2}+\frac{c}{4} \sum \operatorname{tr} A_{\varphi(i)}^{2} \\
& +(1+a) \sum\left(R_{i j k s} h_{i l}^{\alpha} h_{k l}^{\alpha}+R_{i j k l} h_{i l}^{\alpha} h_{j k}^{\alpha}\right) .
\end{aligned}
$$

Now, we need the following algebraic lemma proved in [3]. 
LEMrMa 1. - Let $A$ and $B$ be symmetrio $(n \times n)$-matsices. Then

$$
-\operatorname{tr}(A B-B A)^{2} \leqq 2\left(\operatorname{tr} A^{2}\right)\left(\operatorname{tr} B^{2}\right)
$$

and the equality holds for nonzero matrices $A$ and $B$ if and only if $A$ and $B$ can be transformed by an orthogonal matrix simultaneously into scalar multiples of $\bar{A}$ and $\bar{B}$, respectively, where

$$
\bar{A}=\left[\begin{array}{ll|l}
0 & 1 & \\
1 & 0 & 0 \\
\hline & 0 & 0
\end{array}\right] \quad \bar{B}=\left[\begin{array}{cc|c}
1 & 0 & 0 \\
0 & -1 & 0 \\
\hline & 0 & 0
\end{array}\right]
$$

Moreover, if $A_{1}, A_{2}, A_{3}$ are symmetric $(n \times n)$-matrices such that

$$
-\operatorname{tr}\left(A_{a} A_{b}-A_{b} A_{a b}\right)^{2}=2\left(\operatorname{tr} A_{a}^{2}\right)\left(\operatorname{tr} A_{b}^{2}\right), \quad 1 \leqq a, b \leqq 3, a \neq b,
$$

then at least one of the matrices $A_{a}$ must be zero.

Now, we shall prove the following.

THEOREM $3 .-$ Let $N$ be an $n$-dimensional compact totally real minimal submanifold of the quatemion projective space $P^{m}(\boldsymbol{H})$. If

$$
\varrho \geqq \frac{n[(n-1)(2 p-1)-p] c}{4(2 p-1)} \quad \text { or equivalently }\|\sigma\|^{2} \leqq \frac{n e}{4(2-1 / p)}
$$

where $p=4 m-n$, then $\mathcal{N}$ is totally geodesic.

Proof. - By (3.8) and Lemma 1, we have

$$
\frac{1}{2} \Delta\|\sigma\|^{2} \geqq\|\bar{\nabla} \sigma\|^{2}-2 \sum_{\alpha \neq \beta}\left(\operatorname{tr} A_{\alpha}^{2}\right)\left(\operatorname{tr} A_{\beta}^{2}\right)-\sum\left(\operatorname{tr} A_{\alpha} A_{\beta}\right)^{2}+\frac{n c}{4}\|\sigma\|^{2}+\frac{o}{4} \sum_{i, p}\left(\operatorname{tr} A_{\varphi(i)}^{2}\right)
$$

Let $S_{\alpha \beta}=\sum \operatorname{tr}\left(A_{\alpha} A_{\beta}\right)=\sum h_{i j}^{\alpha} h_{j i}^{\beta}$. Then $\left(S_{\alpha \beta}\right)$ is a symmetric $(p \times p)$ matrix and it can be diagonalized for a suitable choice of $\left\{e_{\alpha}\right\}$. Hence we may assume that $\operatorname{tr} A_{\alpha} A_{\beta}=0$ for $\alpha \neq \beta$. Furthermore, if we put

$$
p \sigma_{1}=\sum \operatorname{tr} A_{\alpha}^{2}=\|\sigma\|^{2}, \quad p(p-1) \sigma_{2}=2 \sum_{\alpha<\beta} \operatorname{tr} A_{\alpha}^{2} \operatorname{tr} A_{\beta}^{2}
$$

we then have

$$
\begin{aligned}
\frac{1}{2} \Delta\|\sigma\|^{2} & =\|\bar{\nabla} \sigma\|^{2}-p(p-1) \sigma_{2}-p^{2} \sigma_{1}^{2}+\frac{n c}{4}\|\sigma\|^{2}+\frac{c}{4} \sum\left(\operatorname{tr} A_{\varphi(i)}^{2}\right) \\
& =\|\bar{\nabla} \sigma\|^{2}+p(p-1)\left(\sigma_{1}^{2}-\sigma_{2}\right)-\left(2 p^{2}-p\right) \sigma_{1}^{2}+\frac{n c}{4}\|\sigma\|^{2}+\frac{c}{4} \sum\left(\operatorname{tr} A_{\varphi(i)}^{2}\right) \\
& =\|\bar{\nabla} \sigma\|^{2}+p(p-1)\left(\sigma_{1}^{2}-\sigma_{2}\right)-\left(2-\frac{1}{p}\right)\|\sigma\|^{4}+\frac{n c}{4}\|\sigma\|^{2}+\frac{c}{4} \sum\left(\operatorname{tr} A_{\varphi(i)}^{2}\right)
\end{aligned}
$$


Since

(3.10) implies

$$
\begin{aligned}
p(p-1)\left(\sigma_{1}^{2}-\sigma_{2}\right) & =(p-1)\left(\sum \operatorname{tr} A_{\alpha}^{2}\right)-2 p \sum_{\alpha<\beta} \operatorname{tr} A_{\alpha}^{2} \operatorname{tr} A_{\beta}^{2} \\
& =\sum_{\alpha<\beta}\left(\operatorname{tr} A_{\alpha}^{2}-\operatorname{tr} A_{\beta}^{2}\right)^{2} \geqq 0
\end{aligned}
$$

$$
\frac{1}{2} \Delta\|\sigma\|^{2} \geqq \frac{n c}{4}\|\sigma\|^{2}-\left(2-\frac{1}{p}\right)\|\sigma\|^{4}=\left[\frac{n c}{4}-\left(2-\frac{1}{p}\right)\|\sigma\|^{2}\right]\|\sigma\|^{2} .
$$

Hence, if $\|\sigma\|^{2} \leqq n c / 4(2-1 / p)$, then $\Delta\|\sigma\|^{2} \geqq 0$. Thus, by a well-known lemma of $\mathrm{E}$. Hopf, we have $\Delta\|\sigma\|^{2}=0$, consequently we have either $\|\sigma\|=0$, i.e. $N$ is totally geodesic or $\|\sigma\|^{2}=n c /(4(2-1 / p))$.

If $\|\sigma\|^{2}=n c /(4(2-1 / p))$, all inequality signs above become equalities. Especially, we have

$$
-\operatorname{tr}\left(A_{\alpha} A_{\beta}-A_{\beta} A_{\alpha}\right)^{2}=2\left(\operatorname{tr} A_{\alpha}^{2}\right)\left(\operatorname{tr} A_{\beta}^{2}\right) .
$$

Thus Lemma 1 implies that at most two of the $A_{\alpha}$ 's are nonzero. Now, in the equality

$$
\sum_{\alpha<\beta}\left(\operatorname{tr} A_{\alpha}^{2}-\operatorname{tr} A_{\beta}^{2}\right)=0,
$$

there are more than three terms in the summation, we have that all $A_{\alpha}$ should be zero. This leads to a contradiction.

If $m=n$, then $\sum_{p, i} \operatorname{tr} A_{\varphi(i)}^{2}=\|\sigma\|^{2}$ and $p=3 n$. Thus (3.10) gives

$$
\frac{1}{2} \Delta\|\sigma\|^{2} \leqq \frac{(n+1) e}{4}\|\sigma\|^{2}-\frac{6 n-1}{3 n}\|\sigma\|^{4} .
$$

By using this inequality and a similar argument as given in the proof of Theorem 3 , we obtain the following.

THeorem 4. - Let $N$ be an n-dimensional compact totally real minimal submanifold of the quaternion projective space $\boldsymbol{P}^{n}(\boldsymbol{H})$. If

$$
\varrho \leqq \frac{n\left(3 n^{2}-5 n-1\right)}{2(6 n-1)} c \quad \text { or equivalently }\|\sigma\|^{2} \leqq \frac{3 n(n+1) c}{4(6 n-1)}
$$

then $N$ is totally geodesic and $\varrho=\frac{1}{4} n(n-1) c$.

\section{4. - Pinehings on sectional curvature.}

In this section we shall use (3.9) and a method in [7] to obtain a sufficient condition for a totally real minimal submanifold in $\boldsymbol{P}^{m}(\boldsymbol{H})$ to be totally geodesic in terms of sectional curvature.

13 - Annali di Matematica 
Let $\alpha_{1}, \ldots, \alpha_{n}$ be the eigenvalues of $A_{\alpha}$. Then we have

$$
\sum_{i, j, k, l}\left(R_{i j k j} h_{i l}^{\alpha} h_{k l}^{\alpha}+R_{i j k l} h_{i l}^{\alpha} h_{j k}^{\alpha}\right)=\frac{1}{2} \sum_{i, k}\left(\alpha_{i}-\alpha_{k}\right)^{2} R_{i k i k}
$$

Now, we assume that the sectional curvature of $N$ is greater than or equal to $\gamma$. (4.1) gives

$$
\sum_{i, j, k, l}\left(R_{i j k j} h_{i l}^{a} h_{k l}^{\alpha}+R_{i j k l} h_{i l}^{\alpha} h_{j k}^{\alpha}\right) \geqq \frac{1}{2} \sum_{i, k}\left(a_{i}-\alpha_{k}\right)^{2} \gamma
$$

Since $N$ is minimal, $\sum_{i, k}\left(\alpha_{i}-\alpha_{k i}\right)^{2}=2 n \operatorname{tr} A_{\alpha}^{2}$. Thus by (3.9) and (4.2) we find

$$
\begin{aligned}
\frac{1}{2} \Delta\|\sigma\|^{2} \geqq\|\bar{\nabla} \sigma\|^{2}+\frac{1-a}{2} \sum \operatorname{tr}\left(A_{\alpha} A_{\beta}-A_{\beta} A_{\alpha}\right)^{2}+a\left(\sum \operatorname{tr} A_{\alpha} A_{\beta}\right)^{2} & \\
& -\frac{n a c}{4}\|\sigma\|^{2}+\frac{e}{4} \sum \operatorname{tr} A_{\varphi(i)}^{2}+(1+a) n \gamma\|\sigma\|^{2}
\end{aligned}
$$

for $a \geqq-1$. By using the arguments as before, we may assume that $\operatorname{tr} A_{\alpha} A_{\beta}=0$ for $\alpha \neq \beta$. Thus by using Lemma 1 again, we get

$$
\begin{aligned}
\frac{1}{2} \Delta\|\sigma\|^{2} \geqq(a-1) \sum_{\alpha \neq \beta}\left(\operatorname{tr} A_{\alpha}^{2}\right)\left(\operatorname{tr} A_{\beta}^{2}\right)+a\left(\sum \operatorname{tr} A_{\alpha}^{2}\right)^{2}-\frac{n a c}{4}\|\sigma\|^{2} & \\
& +\frac{c}{4} \sum \operatorname{tr} A_{\varphi(j)}^{2}+(1+a) n \gamma\|\sigma\|^{2} .
\end{aligned}
$$

Since $\sum_{\alpha \neq \beta}\left(\operatorname{tr} A_{\alpha}^{2}\right)\left(\operatorname{tr} A_{\beta}^{2}\right)+\sum_{\alpha}\left(\operatorname{tr} A_{\alpha}^{2}\right)^{2}=\left(\sum_{\alpha} \operatorname{tr} A_{\alpha}^{2}\right)^{2}=\|\sigma\|^{4}$ and $\sum\left(\operatorname{tr} A_{\alpha}^{2}\right)^{2} \geqq\|\sigma\|^{4} / n,(4.4)$ gives

$$
\frac{1}{2} \Delta\|\sigma\|^{2} \geqq\left\{\frac{1}{n}-(1-a)\right\}\|\sigma\|^{4}+\left\{(1+a) n \gamma-\frac{n a c}{4}\right\}\|\sigma\|^{2}+\frac{c}{4} \sum \operatorname{tr} A_{\varphi(i)}^{2} .
$$

In particular, putting $a=1-1 / n$, we obtain

$$
\frac{1}{2} \Delta\|\sigma\|^{2} \geqq\left\{(2 n-1) \gamma-\frac{n-1}{4} e\right\}\|\sigma\|^{2}+\frac{e}{4} \sum \operatorname{tr} A_{\varphi(i)}^{2} .
$$

Therefore, if $\gamma \geqq(n-1) o / 4(2 n-1)$, then the right-hand side of $(4.5)$ is nonnegative. Thus, by using Hopf's lemma again, we obtain $\Delta\|\sigma\|^{2}=0, \sum \operatorname{tr} A_{\varphi(i)}^{2}=0$ and the inequality signs in this section are actually equalities. Thus, in particular, we have

$$
-\operatorname{tr}\left(A_{\alpha} A_{\beta}-A_{\beta} A_{\alpha}\right)^{2}=2\left(\operatorname{tr} A_{\alpha}^{2}\right)\left(\operatorname{tr} A_{\beta}^{2}\right)
$$

for $\alpha \neq \beta$. Thus at most two of the $A_{\alpha}$ 's are nonzero and, with respect to suitable 
frame, these two matrices are scalar multiples of $\bar{A}$ and $\bar{B}$ in lemma 1 . Without loss of generality, we may assume that

$$
\begin{gathered}
A_{n+1}=a\left(\begin{array}{ll|l}
0 & 1 & 0 \\
1 & 0 & 0 \\
\hline 0 & 0
\end{array}\right), \quad A_{n+2}=b\left(\begin{array}{rr|r}
1 & 0 & 0 \\
0 & -1 & 0 \\
\hline 0 & 0
\end{array}\right) \\
A_{\alpha}=0 \quad \text { for } \alpha \neq n+1, n+2 .
\end{gathered}
$$

If $N$ is not totally geodesic in $\boldsymbol{P}^{m}(\boldsymbol{H})$, then $\gamma=(n-1) 0 / 4(2 n-1)$. On the other hand, by (2.4)' and (4.6), we find that the sectional curvature $K\left(\pi\left(e_{1}, e_{i}\right)\right)$ of the plane $\pi\left(e_{1}, e_{i}\right)$ satisfies

$$
K\left(\pi\left(e_{1}, e_{i}\right)\right)=R_{1 i 1 i}=c / 4 \quad \text { for } i>2 .
$$

Since $N$ is not totally geodesic, we may assume that $A_{n+2} \neq 0$. For $A_{\alpha}=A_{n+2}$, we have $\alpha_{1}=b, \alpha_{2}=-b, \alpha_{i}=0, i>2$. Thus $\left(\alpha_{1}-\alpha_{i}\right)^{2} \neq 0$ for $i>2$. Therefore, from (4.1) and the equality of (4.2), we find $R_{1 i 1 i}=\gamma=(n-1) e / 4(2 n-1)$ which is smaller than $c / 4$. Consequently, we have $n=2$. Since $\sum \operatorname{tr} A_{\varphi(i)}^{2}=0, m>2$ and $K=c / 12$.

If $n=m$, then $\sum \operatorname{tr} A_{\varphi(i)}^{2}=\|\sigma\|^{2}$. Thus (4.5) reduces to

$$
\frac{1}{2} \Delta\|\sigma\|^{2} \geqq\left\{(2 n-1) \gamma-\frac{n-2}{4} c\right\}\|\sigma\|^{2} .
$$

Thus if $\gamma \geqq(n-2) o / 4(2 n-1)$, then $\Delta\|\sigma\|^{2}=0$. By a similar argument, we may prove that either $N$ is totally geodesic or $n=m=2$ and $K=0$. Consequently, we have the following.

THEOREM 5. - Let $N$ be an $n$-dimensional compact totally real minimal submanifold of the quaternion projective space $P^{m}(\boldsymbol{H})$. If the sectional curvature of $N$ satisfies

$$
K \geqq \frac{(n-1) c}{4(2 n-1)},
$$

hen either (i) $N$ is totally geodesic in $P^{m}(\boldsymbol{H})$ or (ii) $n=2, m>2$ and $N$ is of positive constant curvature o/12.

THEOREM $6 .-$ Let $N$ be an $n$-dimensional compact, totally real, minimal submanifold of the quaternion projective space $P^{n}(\boldsymbol{H})$. If the sectional curvature of $N$ satisfies

$$
K \geqq \frac{(n-2) c}{4(2 n-1)},
$$

then either (i) $N$ is totally geodesio in $P^{n}(\boldsymbol{H})$ or (ii) $n=2$ and $N$ is a flat surface. 
REMARK 2. - Let $(x, y, z)$ be the natural coordinate system in $\boldsymbol{R}^{3}$ and $\left(u^{1}, u^{2}, u^{3}\right.$ $\left.u^{4}, u^{5}\right)$ the natural coordinate system in $\boldsymbol{R}^{5}$. We consider the mapping defined by

$$
\begin{aligned}
& u^{1}=\frac{1}{\sqrt{3}} y z, \quad u^{2}=\frac{1}{\sqrt{3}} x y, \quad u^{3}=\frac{1}{\sqrt{3}} x z, \\
& u^{4}=\frac{1}{2 \sqrt{3}}\left(x^{2}-y^{2}\right), \quad u^{5}=\frac{1}{6}\left(x^{2}+y^{2}-2 z^{2}\right) .
\end{aligned}
$$

This defines an imbedding of the real projective plane into the unit hypersphere $S^{4} \subset R^{5}$. This real projective plane in $S^{4}$ is called the Veronese surface. It is a minimal submanifold of $\$^{4}$ with sectional curvature $K=\frac{1}{3}$. Now, we consider the immersion of $S^{4}$ into the real projective space $P^{4}(\boldsymbol{R})$ of sectional curvature 1 and the canonical totally geodesic totally real isometric imbedding of $P^{4}(R)$ into the quaternion projective space $P^{m}(\boldsymbol{H})$ of constant quaternion sectional curvature $4=c$. Then the composition immersion of the Veronese surface into $P^{m}(\boldsymbol{H})$ gives us a compact, totally real, minimal surface in $P^{4}(\tilde{H})$ with $K=c / 12$ and $\bar{\nabla} \sigma=0$.

Remark 3. - Let $T^{2}$ be a flat, totally real, minimal surface in the complex projective plane $P^{2}(C)$ of constant holomorphic sectional curvature $c$. Then since $P^{2}(C)$ can be isometrically imbedded in $P^{2}(\boldsymbol{H})$ as a totally geodesic, totally real submanifold, the composition immersion gives us a compact totally real minimal surface in $P^{2}(\boldsymbol{H})$ with $K=0$ and $\bar{\nabla} \sigma=0$.

From Remarks 2 and 3, we see that Theorems 5 and 6 are best possible. By using similar arguments as given in [3], we may prove that the Veronese surface in $P^{4}(\boldsymbol{H})$ is the only compact totally real minimal submanifold in $P^{4}(\boldsymbol{H})$ with $K=$ $=((n-1) / 4(2 n-1)) e$.

THEOREM 7. - Let $N$ be an $n$-dimensional compact totally real minimal submanifold of the complex projective space $P^{m}(C)$ of constant holomorphic sectional curvature $c$. If the sectional curvature of $N$ satisfies

$$
K \geqq \frac{(n-1) c}{4(2 n-1)},
$$

then either (i) $N$ is totally geodesic in $P^{m}(C)$ or (ii) $n=2, m>2$ and $N$ is of positive constant curvature c/12.

This theorem follows from Theorem 5 and the fact that $N \subset P^{m}(C) \subset P^{m}(H)$ can be regarded as a totally real minimal submanifold of $\boldsymbol{P}^{m}(\boldsymbol{H})$.

REMARK 4. - If we use the similar argument as in [3], we may prove that $N$ is in fact the Veronese surface given in Remark 2.

Similarly, by using Theorem 6, we have the following. 
THEOREM 8. - Let $N$ be an $n$-dimensional compact totally real minimal submanitold of the complex projective space $P^{n}(C)$ of constant holomorphic sectionat ourvature $c$. If we have

$$
K \geqq \frac{(n-2) e}{4(2 n-1)},
$$

then either (i) $N$ is totally geodesio in $P^{n}(C)$ or (ii) $n=2$ and $N$ is a flat surface.

Theorem 8 improves slightly the main theorem of [5].

REMARK 5. - It shall be noticed that Theorems 5 and 7 are independent of codimension of $N$ in the ambient spaces.

\section{5. - Real-space-forms in quaternion-space-forms.}

In this section, we shall assume that $N$ is of constant sectional curvature $c^{l}$ isometrically immersed in a $4 n$-dimensional quaternion-space-form $M^{m}(c)$ of quaternion sectional curvature $c$.

LEMMA 2. - Let $N$ be an n-dimensional totally real minimal submanifold in a quaternion-space-form $M^{n}(c)$. If $N$ has constant sectional curvature $e^{\prime}$, then

$$
\begin{gathered}
\operatorname{tr}\left(\sum_{\varphi, i} A_{\varphi(i)}^{2}\right)^{2}=\sum_{\varphi, i, j}\left(\operatorname{tr} A_{\varphi(i)} A_{\varphi(j)}\right)^{2}, \\
c^{\prime} \leqq c / 4 \\
\sum_{\varphi} \operatorname{tr}\left(A_{\varphi(i)} A_{\varphi(j)}\right)=\|\sigma\|^{2} \frac{\delta_{i j}}{n}, \\
\sum_{\varphi, i, j} \operatorname{tr}\left(A_{\varphi(i)} A_{\varphi(j)}\right)^{2}-\sum_{\varphi, i, j}\left(\operatorname{tr} A_{\varphi(i)} A_{\varphi(j)}\right)^{2}=\left(c^{\prime}-\frac{c}{4}\right)\|\sigma\|^{2} .
\end{gathered}
$$

Proof. - (1) From (2.9), we have

$$
\begin{aligned}
\operatorname{tr}\left(\sum A_{\varphi(i)}^{2}\right)^{2} & =\sum \operatorname{tr} A_{\varphi(i)}^{2} A_{\varphi(j)}^{2}=\sum h_{k l}^{\varphi(i)} h_{l m}^{\varphi(i)} h_{m h}^{\varphi(j)} h_{h k}^{\varphi(j)} \\
& =\sum h_{i l}^{\varphi(k)} h_{l i}^{\varphi(m)} h_{j h}^{\varphi(m)} h_{h i}^{\varphi(k)}=\sum\left(\operatorname{tr} A_{\varphi(k)} A_{\varphi(m)}\right)^{2}
\end{aligned}
$$

(2) Let the scalar curvature of $N$ be $\varrho$. Then $\varrho=n(n-1) e^{\prime}$. Thus

$$
n(n-1) e^{\prime}=\frac{1}{4} n(n-1) e-\|\sigma\|^{2} \leqq \frac{1}{4} n(n-1) e .
$$

This implies $e^{\prime} \leqq o / 4$. 
(3) Since $N$ is of constant sectional curvature $e^{\prime} . N$ is Einsteinian and the Ricei tensor $S$ of $\dot{N}$ is $S=\varrho g / n$. Thus by the equation of Gauss, we find

$$
\sum A_{\varphi(j)}^{2}=\left(\frac{1}{4}(n-1) e-\frac{\varrho}{n}\right) E=\frac{\|\sigma\|^{2}}{n} E,
$$

when $E$ is the identity transformation. Hence

$$
\frac{\|\sigma\|^{2}}{n} \delta_{i j}=\sum h_{i l}^{\varphi(k)} h_{i j}^{\varphi(k)}=\sum h_{i k}^{\varphi(i)} h_{i l}^{\varphi(j)}=\sum_{\varphi} \operatorname{tr}\left(A_{\varphi(i)} A_{\varphi(j)}\right) .
$$

(4) The curvature tensor of $N$ satisfies

$$
R_{i j k l}=e^{\prime}\left(\delta_{i k} \delta_{j b}-\delta_{i l} \delta_{j k}\right) \text {. }
$$

On the other hand, the curvature tensor $\tilde{R}$ of $M$ satisfies

$$
\tilde{R}_{i j k l}=\frac{c}{4}\left(\delta_{i l k} \delta_{j l}-\delta_{i l} \delta_{j k}\right)
$$

along $N$. Thus by the equation of Gauss, we have

$$
\sum\left(h_{i k}^{\varphi(m)} h_{j l}^{\varphi(m)}-h_{i l}^{\varphi(m)} h_{j k}^{\varphi(m)}\right)=\left(c^{\prime}-\frac{e}{4}\right)\left(\delta_{i k} \delta_{j l}-\delta_{i l} \delta_{j k}\right)
$$

Multiplying $\sum h_{i l}^{\varphi(r)} h_{i k}^{\varphi(r)}$ on both sides and sum up, we get (4).

THeorem 9. - Let $N$ be an n-dimensional totally real minimal submanifold in the 4n-dimensional quaternion projective space $P^{n}(\boldsymbol{H})$. If the sectional curvature of $N$ is constant, say $c^{\prime}$, then either (i) $N$ is totally geodesic, (i.e., $e^{\prime}=o / 4$ ) or (ii) $N$ is non-positively curved, i.e., $c^{\prime} \leqq 0$.

Proof. - If $N$ has constant sectional curvature $c^{\prime}$, then its scalar curvature $\varrho=$ $=n(n-1) o^{\prime}$ is also constant. Thus $\|\sigma\|^{2}=\frac{1}{4} n(n-1) c-\varrho$ is constant, too. By (3.8) we find

$$
0=\|\bar{\nabla} \sigma\|^{2}+\sum \operatorname{tr}\left(A_{\alpha} A_{\beta}-A_{\beta} A_{\alpha}\right)^{2}-\sum\left(\operatorname{tr} A_{\alpha} A_{\beta}\right)^{2}+\frac{(n+1) e}{4}\|\sigma\|^{2} .
$$

Notice that $\alpha, \beta, \ldots$ run on $\varphi(1), \ldots, \varphi(n)$ for $\varphi=I, J, K$. From these we get

$$
\begin{aligned}
0 & =\|\bar{\nabla} \sigma\|^{2}+2 \sum \operatorname{tr}\left(A_{\varphi(i)} A_{\varphi(j)}\right)^{2}-2 \sum\left(\operatorname{tr} A_{\varphi(i)}^{2} A_{\varphi(j)}^{2}\right)-\sum\left(\operatorname{tr} A_{\varphi(i)} A_{\varphi(j)}\right)^{2}+\frac{(n+1) e}{4}\|\sigma\|^{2} \\
& =\|\bar{\nabla} \sigma\|^{2}+2 \sum \operatorname{tr}\left(A_{\varphi(i)} A_{\varphi(j)}\right)^{2}-2 \operatorname{tr}\left(\sum A_{\varphi(i)}^{2}\right)^{2}-\sum\left(\operatorname{tr} A_{\varphi(i)} A_{\varphi(i)}\right)^{2}+\frac{(n+1) e}{4}\|\sigma\|^{2}
\end{aligned}
$$

As in the proof of our previous theorems, we may assume that $\operatorname{tr} A_{\varphi(i)} A_{\varphi(j)}=0$ for $i \neq j$. Using Lemma 2 , we have

$$
0=\|\bar{\nabla} \sigma\|^{2}+2 \sum \operatorname{tr}\left(A_{\varphi(i)} A_{\varphi(i)}\right)^{2}-3 \operatorname{tr}\left(\sum A_{\varphi(i)}^{2}\right)^{2}+\frac{(n+1) c}{4}\|\sigma\|^{2} .
$$


Substituting (3) and (4) of Lemma 2 into this equation, we find

$$
\begin{aligned}
\sum \operatorname{tr}\left(A_{\varphi(i)} A_{\varphi(j)}\right)^{2} & =\sum\left(\operatorname{tr} A_{\varphi(i)} A_{\varphi(j)}\right)^{2}+\left(c^{\prime}-\frac{c}{4}\right)\|\sigma\|^{2} \\
& =\sum\left(\operatorname{tr} A_{\varphi(i)}^{2}\right)^{2}+\left(c^{\prime}-\frac{e}{4}\right)\|\sigma\|^{2} \\
& =\frac{\|\sigma\|^{4}}{n}+\left(c^{\prime}-\frac{c}{4}\right)\|\sigma\|^{2} .
\end{aligned}
$$

Thus, we have

$$
0=\|\bar{\nabla} \sigma\|^{2}+\frac{(n-1) e+8 c^{\prime}}{4}\|\sigma\|^{2}-\frac{\|\sigma\|^{4}}{n}
$$

Combining this with $\|\sigma\|^{2}=n(n-1)\left(c / 4-e^{\prime}\right)$, we get

$$
\|\bar{\nabla} \sigma\|^{2}=n\left(n^{2}-1\right) e^{\prime}\left(c^{\prime}-\frac{e}{4}\right) \text {. }
$$

From this and (2) of Lemma 2, we deduce that $e^{\prime}=c / 4$ or $e^{\prime} \leqq 0$.

The following result is an immediate consequence of Theorem 9.

THEOREM 10. - Let $N$ be an n-dimensional totally real minimal submanifold in the 4n-dimensional quaternion projective space $P^{n}(\boldsymbol{H})$. If (1) the sectional curvature of $N$ is constant and (2) the second fundamental form $\sigma$ is parallel, then either (i) $N$ is totally geodesic or (ii) $N$ is flast.

RuRMARK 6. - Since the Veronese surface in $P^{4}(\boldsymbol{H})$ is a minimal totally real surface of constant curvature $c / 12$ with parallel second fundamental form. Theorems 9 and 10 are not true for arbitrary codimension.

\section{REFERENCES}

[1] B. Y. CHEN, Totally umbilical submanifolds of quaternon-space-forms, J. Austra. Math. Soc., 26 (1978), pp. 154-162.

[2] B. Y. Chen - K. OGrue, On totally real submanifolds, Trans, Amer. Math. Soc., 193 (1974), pp. $257-266$.

[3] S. S. CheRn - M. P. Do CARMo - S. Kobaxashi, Minimal submanifolds of a sphere with second fundamental form of constant length, Funetional Analysis and Related Fields, in Proc. Conf. for M. Stone, Univ. of Chicago, 1968, Springer, New York (1970), pp. 59-75.

[4] S. Isminara, Quaternion Kaehlerian manifolds, J. Differential Geometry, 9 (1974), pp. 483-500.

[5] K. OGIus, Positively curved totally real minimal submanifolds immersed in a complex projective space, Proc. Amer. Math. Soc., 56 (1976), pp. 264-266.

[6] K. Yavo - M. KoN, Anti-invariant submanifolds, M. Dekker, New York, 1976.

[7] S. T. YAU, Submanifolds with constant mean curvature, II, Amer. J. Math., 97 (1975), pp. $76-100$. 